\title{
Small-signal analysis of naturally-sampled single-edge PWM control loops
}

\author{
Toit Mouton*, Stephen $\operatorname{Cox}^{\dagger}$, Brendan McGrath ${ }^{\ddagger}$, Lars Risbo ${ }^{\S}$ and Bruno Putzeys $₫$ \\ * Department of Electrical and Electronic Engineering \\ University of Stellenbosch, Private Bag X1, Matieland, 7602, South Africa \\ Email: dtmouton@sun.ac.za
}

$\dagger$ School of Mathematical Sciences, University of Nottingham, University Park, Nottingham NG7 2RD, United Kingdom

Email: stephen.cox@nottingham.ac.uk

\author{
${ }^{\ddagger}$ School of Electrical and Computer Engineering, RMIT University, Melbourne, Australia, \\ Email: brendan.mcgrath@rmit.edu.au \\ $\S$ Purifi ApS, Hørhavevej 66A, 8270 Højbjerg, Denmark, Email: lars@ purifi.dk \\ ฯ Purifi ApS, Hørhavevej 66A, 8270 Højbjerg, Denmark, Email: bruno.putzeys@gmail.com
}

\begin{abstract}
This paper presents a simple method to analyse the behaviour of feedback loops that contain a naturally-sampled single-edge pulse-width modulator. A small-signal model is derived by means of simple geometric arguments. It is shown how this small-signal model can be used to analyse the stability of the continuous-time pulse-width modulated feedback loop by using standard $z$-domain techniques. The strategy relies on familiar concepts like transfer functions and small-signal gains and does not require any in-depth knowledge of non-linear systems. A simple design process, where the continuous-time compensator is designed directly in the $z$-domain, is developed and detailed design equations are derived for a PI current regulator. It is shown how the proposed strategy can accurately predict instability that cannot be explained by means of the well-known average model of the pulse-width modulator. The theoretical analysis is confirmed by means of detailed time-domain simulations. The mechanisms that lead to instability are discussed and an equation for the critical loop gain is derived.
\end{abstract}

\section{Index Terms}

Small-signal model, pulse-width modulation, stability, bifurcation 


\section{INTRODUCTION}

The theory of the stability of pulse-width modulated control loops has been studied for more than half a century [1]-[6] and is almost as old as the field of Power Electronics itself. The most widely used method to derive a transfer function for a pulse-width modulated converter is the state-space averaging technique, introduced by Middlebrook and Cuk in [7]. The main advantages of state-space averaging are its simplicity and the fact that it can easily be applied to design problems by using techniques from classical linear control theory. One of the major disadvantages of this technique is the fact that it fails to predict the stability of even the simplest PWM feedback loops correctly [8].

Another technique that has gained popularity is generalised state-space averaging, which was introduced in [9]. According to this method, the circuit's state variables are approximated by a Fourier series of which the coefficients are time dependent. The greater the order of the harmonics considered in the model, the more accurate the model will become. This method was extended to include feedback in [10] and has since been the subject of intense research [11]-[14].

Over the years a vast number of different approaches have been developed to analyse the non-linear behaviour of power electronic converters. A complete summary of all these approaches would result in a substantial comprehensive survey paper and falls beyond the scope of the current paper. The following paragraphs provide an overview of the use of discrete-time small-signal models to analyse the behaviour of continuous-time PWM control loops.

The first comprehensive study of the use of discrete-time models to analyse the small-signal behaviour of power electronic converters can be found in [15]. The basis for this work is to utilise straight-line approximations to study the steady-state behaviour of the converter. In [16] the full state-space equations that describe the non-linear system are derived. The steady-state operating point is determined through simulation, after which the system is linearised to produce a small-signal model. The eigenvalues of the linearised model are used to analyse the stability of the converter. In [17] a linear small-signal model is derived which gives rise to a discrete-time modelling approach, based on the state space-representation of the converter. A general approach to sampled-data modelling of power electronic converters is described in [18]. This approach requires the use of symbolic computer manipulation to calculate the matrix exponentials that arise when modelling the system. It then makes use of multi-variable Taylor series expansions to derive the small-signal model. The approach followed in [19], [20] is broadly similar to that of [16]. The differential equations that describe the full non-linear system are derived and the steady-state operating point is determined through simulation. Standard mathematical linearisation techniques are then applied to derive a linear sampled-data model. A unified framework for modelling closed-loop PWM DC-to-DC converters based on concepts from Banach spaces and the time-lifting technique is presented in [21]. In [22] previously developed sampled-data modelling techniques of [9], [15], [17] are used to obtain a discrete-time model of a dual active-bridge converter.

The idea of using discrete-time models to analyse the behaviour of continuous-time pulse-width modulated control loops developed independently in the class-d audio community. It is imperative to have accurate models 
when designing high-performance class-d amplifiers since very high loop gains are required to obtain a flat frequency response over the audio band, low output impedance and sufficient noise and error suppression. In [23] a small-signal model that leads to a discrete-time model is derived within the context of generalised carriers with double-edge modulation. While the small-signal model of [23] is derived in a similar way to that of [17] the small-signal model of [23] incorporates the effect of the ripple gradient into the small-signal model itself. Applications of this model are described in [24] and [25]. It should be pointed out that the discrete-time analysis of the double-edge modulator in [23] is accurate only near a steady-state operating point with a 50\% duty cycle idle point. In [26] a discrete-time model of a class-d amplifier that incorporates the ripple compensation technique of [27] was derived and used to analyse the steady-state response, the stability of the steady-state solution and to derive a small-signal model as well as a large-signal model of the loop.

In this paper is shown how the small-signal behaviour of a continuous-time control loop, containing a naturallysampled single-edge pulse-width modulator, can be studied by using well-known techniques from classical control theory. A small-signal model is derived, according to which the pulse-width modulator is modelled as an ideal sampler and an impulse generator. It is shown that the small-signal gain of the pulse-width modulator depends on the ripple gradient of the input signal to the pulse-width modulator. It is subsequently shown how the continuoustime feedback loop can be transformed to the $z$-domain where standard $z$-domain methods can be used to analyse its stability. A major advantage of this approach is that it leads to a simple $z$-domain design strategy which yields the coefficients of the continuous-time controller. Detailed design equations are developed for a PI current regulator. An analytic equation for the small-signal gain, which previously had to be determined through time-domain simulations, is derived. The concept of the critical loop gain is introduced and a closed-form solution for the value of the critical loop gain is derived.

The strategy presented here does not require any specialised knowledge of non-linear control systems. It is based on familiar concepts like transfer functions and loop gains and can easily be applied by practising engineers to design high-performance control loops. The methods discussed in this paper provide significant physical insight into the mechanisms that govern the stability of naturally-sampled control loops, thereby demystifying some of the behaviour that cannot be explained by means of the average model.

The work discussed in this paper is not limited to analogue control loops, but is also applicable to highly oversampled digital control loops, in which the sampling frequency is significantly higher than the switching frequency. The design and FPGA implementation of a highly oversampled digital current regulator is discussed in [28].

The small-signal model of the single-edge naturally-sampled pulse-width modulator is derived in section II. In section III it is systematically explained why the small-signal model accurately predicts the stability of the largesignal non-linear feedback loop. The use of $z$-domain methods to model the small-signal feedback loop is discussed in section IV, while an analytic expression for the small-signal gain is derived in section V. New design equations for a PI current regulator are presented in section VI. The theoretical results of the previous sections are compared with time-domain simulations in section VII, which also contains a detailed discussion of the mechanisms that lead 
to instability and an equation for the critical loop gain. Sections II and IV are mainly an adaptation of existing ideas from [17], [23] to a single-edge carrier-based pulse-width modulator. The main difference is that the model of [23] is accurate only around a $50 \%$ duty cycle idling point, while the techniques presented here are accurate for any value of duty cycle. The rest of the paper consists mainly of original work.

\section{SMALL-SigNAL MODEL OF THE SINGLE-EDGE PULSE-WIDTH MODULATOR}

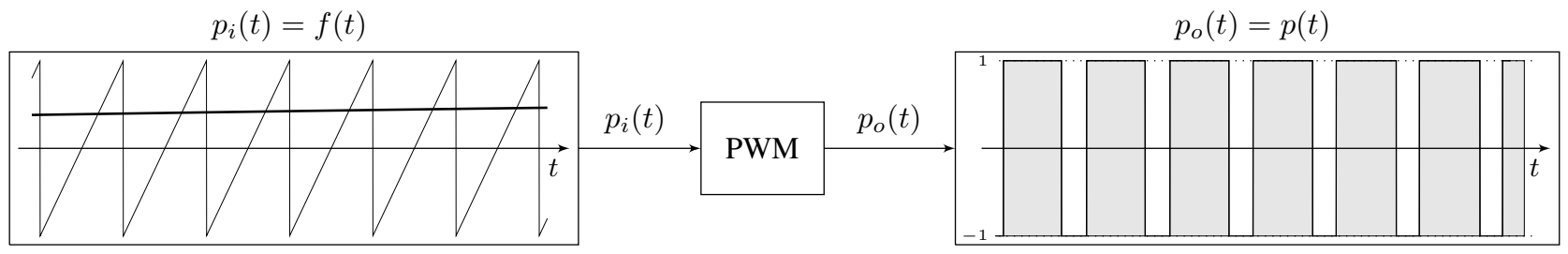

(a) Reference signal $f(t)$ is applied to the input of the modulator. This produces a PWM pulse train denoted by $p(t)$.

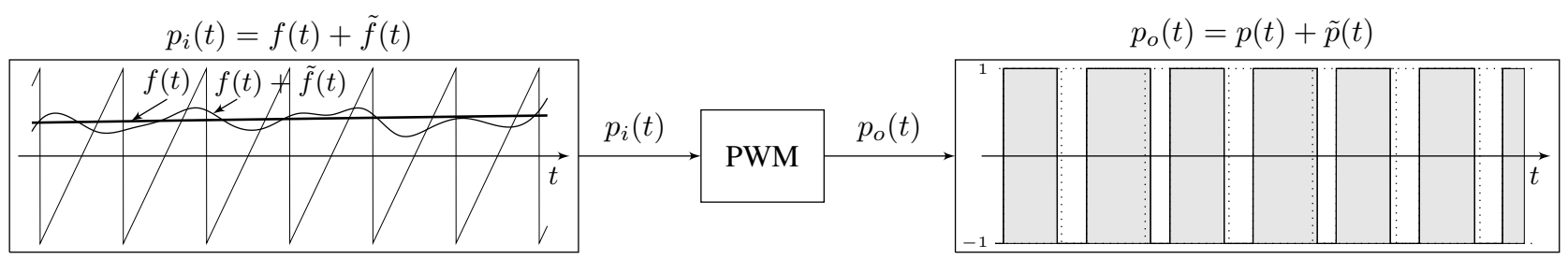

(b) Reference signal $f(t)$ plus a small perturbation signal $\tilde{f}(t)$ is applied to the input of the modulator. This produces a PWM pulse train denoted by $p(t)+\tilde{p}(t)$.

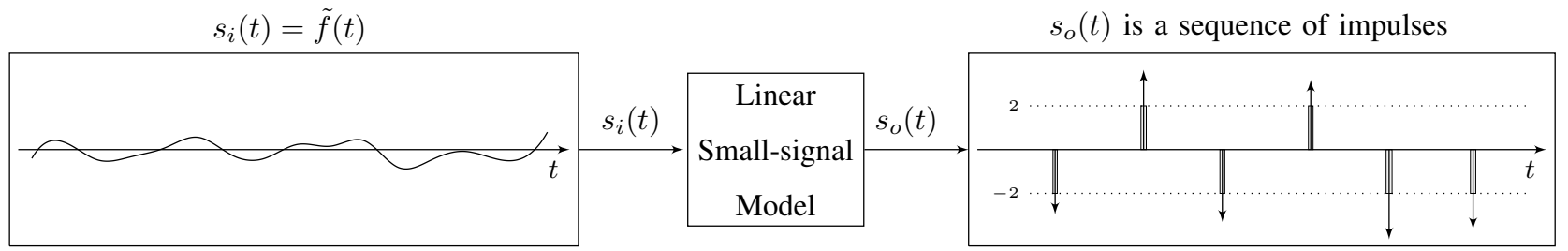

(c) The sequence $\tilde{p}(t)$ of narrow rectangular pulses is the difference between the PWM pulse trains in (a) and (b). This sequence of narrow rectangular pulses is modelled as a sequence of impulses. The strength of each impulse is equal to the area of the narrow rectangular pulse it represents.

Figure 1: Derivation of the small-signal model of the pulse-width modulator.

Numerous ways to analyse the small-signal behaviour of power electronic converters have been developed over the past decades [8], [12], [13], [29]. The small-signal model described in this section is derived by means of simple and intuitive geometric arguments.

In Fig. 1(a) a large signal $f(t)$ is applied to the input $p_{i}(t)$ of the single-edge naturally-sampled pulse-width modulator. The pulse-width modulator compares $f(t)$ to a sawtooth carrier and generates a PWM pulse train denoted by $p(t)$. For the sake of simplicity it is assumed that the amplitude of the carrier is equal to 1 . It is also assumed that the pulse-width modulator contains set-reset logic and will thus only respond to the first intersection between its input signal $f(t)$ and the carrier during each switching period. 
A small perturbation signal $\tilde{f}(t)$ is added to $f(t)$ in Fig. 1(b) and applied to the input of the pulse-width modulator. In comparison with Fig. 1(a), the falling pulse edges in Fig. 1(b) are slightly shifted in time due to this perturbation signal. The resulting PWM pulse train is denoted by $p(t)+\tilde{p}(t)$. The narrow rectangular pulses on the right-hand side of Fig. 1(c) show the difference between $p(t)$ and $p(t)+\tilde{p}(t)$. The height of each rectangular pulse is equal to 2 (i.e. the peak-to-peak amplitude of the PWM pulse train) and its width corresponds to the time shift in the falling pulse edges resulting from $\tilde{f}(t)$. The distance between successive pulses is approximately equal to the switching period $T_{s}$.

It is well known that a sequence of narrow rectangular pulses can be modelled by a sequence of impulses (Dirac delta distributions) [30], as shown in Fig. 1(c). The strength of each impulse is equal to the area of the narrow rectangular pulse it represents. The small-signal model of the pulse-width modulator is a mathematical model that will produce the sequence $\tilde{p}(t)$ of impulses at its output, if its input signal is $\tilde{f}(t)$.

Up to this point the derivation of the small-signal model is almost identical to that of [17]. One major difference is the fact that the bus voltage of the converter is taken as the input signal in [17]. As a result, the strategy presented in [17] can be used to analyze the stability of the loop. However, it cannot be used to derive a closed-loop referenceto-output transfer function of the loop. It will later be shown in Eq. (8) that the approach that is followed in the current paper does lead to a closed-loop small-signal transfer function.

Fig. 2 shows a zoomed view of one of the narrow rectangular pulses. The sampling time $t_{s p}$ is defined as the time instant at which the large signal $f(t)$ intersects the carrier. Given that the gradient of the carrier is equal to $\frac{2}{T_{s}}$, where $T_{s}=\frac{1}{f_{s}}$ is the switching period, then by simple geometrical arguments (or by Taylor-series expansion of the right-hand side of Eq.(1) and truncation at first order in small terms) it is straightforward to show that:

$$
\begin{aligned}
\frac{2}{T_{s}} \Delta T & =f\left(t_{s p}+\Delta T\right)+\tilde{f}\left(t_{s p}+\Delta T\right)-f\left(t_{s p}\right) \\
& \approx f^{\prime}\left(t_{s p}\right) \Delta T+\tilde{f}\left(t_{s p}\right),
\end{aligned}
$$

where $f^{\prime}\left(t_{s p}\right)$ is the derivative of the large-signal modulator input $f(t)$ at the sampling point.

Due to the fact that $\Delta T$ is small, two approximations were made to derive Eq. (2) from Eq. (1). The first approximation is that

$$
\frac{f\left(t_{s p}+\Delta T\right)-f\left(t_{s p}\right)}{\Delta T} \approx f^{\prime}\left(t_{s p}\right) .
$$

The second approximation is that $\tilde{f}\left(t_{s p}+\Delta T\right) \approx \tilde{f}\left(t_{s p}\right)$.

Solving for $\Delta T$ from Eq. (2) yields

$$
\Delta T \approx \frac{\tilde{f}\left(t_{s p}\right)}{2 f_{s}-f^{\prime}\left(t_{s p}\right)} .
$$

Since the the height of the rectangular pulse is equal to 2 , the area $A$ of the pulse is given by:

$$
A \approx \frac{2}{2 f_{s}-f^{\prime}\left(t_{s p}\right)} \tilde{f}\left(t_{s p}\right) .
$$

It is thus evident that the area of each narrow rectangular pulse (and hence the strength of the impulse that represents it) is proportional to the value of the small-signal perturbation $\tilde{f}\left(t_{s p}\right)$, and depends on the derivative of 
the large-signal input $f^{\prime}\left(t_{s p}\right)$ at the sampling point. Eq. (3) can be rewritten as

$$
A \approx \tilde{f}\left(t_{s p}\right) K_{s s} T_{s}
$$

where

$$
K_{s s}=\frac{2 f_{s}}{2 f_{s}-f^{\prime}\left(t_{s p}\right)} .
$$

$K_{s s}$ is called the small-signal gain of the pulse-width modulator. It depends on the switching frequency $f_{s}$ as well as the gradient of the input signal to the pulse-width modulator just prior to its intersection with the carrier. This gradient will subsequently be referred to as the ripple gradient.

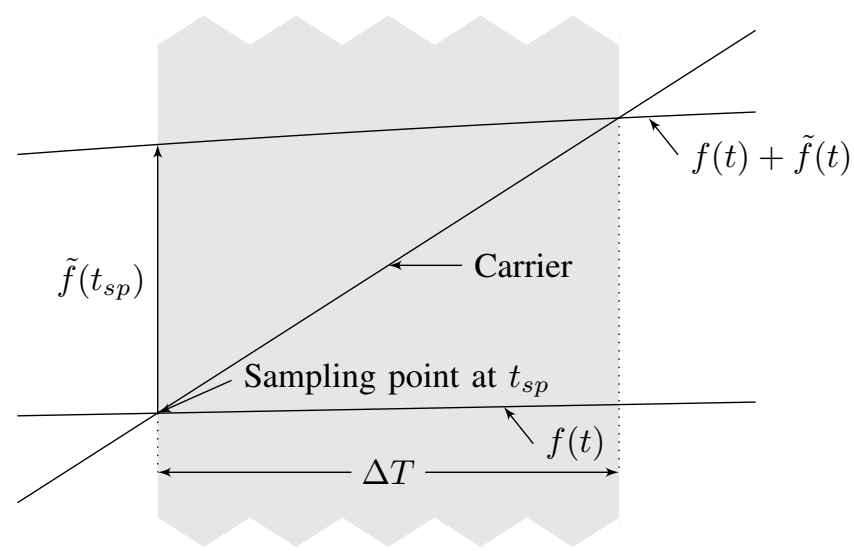

Figure 2: Zoomed view of the perturbed and unperturbed intersection points, leading to a narrow rectangular pulse.

Fig. 3 shows the resulting equivalent block diagram of the PWM small-signal model. It consist of an ideal sampler, which samples its input signal at each intersection of the carrier and the input signal of the pulse-width modulator, i.e. the sampling point. The value of $\tilde{f}\left(t_{s p}\right)$ is multiplied by the small-signal gain $K_{s s}$ and sent to an impulse generator which instantaneously produces an impulse of strength $\tilde{f}\left(t_{s p}\right) K_{s s}$. This impulse is subsequently multiplied by a factor $T_{s}$. This happens once per switching period. The resulting sequence of numbers can be seen as a discrete-time signal which can be analysed by using z-domain methods. This will be discussed in more detail in section IV. The small-signal model of [17] does not incorporate the effect of the ripple gradient into the small-signal model itself. The effect of the ripple gradient has to be incorporated in the so-called effective gain

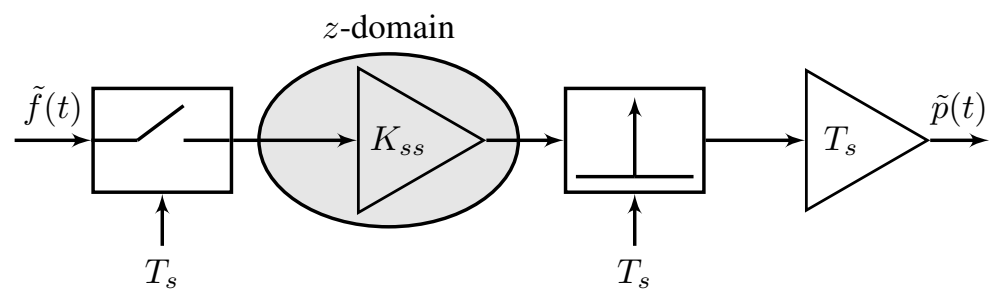

Figure 3: Small-signal model of the single-edge pulse-width modulator. 
vector in [17]. However, it is not explained how to do this for a carrier-based PWM strategy in [17]. The effect of the ripple gradient is quite subtle and can easily be overlooked.

It is important to note that the small-signal model is linear. Furthermore, if $f(t)$ is periodic, so that $f\left(t+T_{s}\right)=f(t)$ for all $t$, then $K_{s s}$ is constant and the sampling becomes strictly uniform. As a result the small-signal model is both linear and time invariant when $f(t)$ is periodic. This assumption of periodicity is used throughout this paper.

Note that $f(t)$ is periodic for systems with a constant reference $i^{\star}(t)$. In this case $f(t)$ consists of a DC component and a periodic ripple component. In [23] a similar small-signal model was derived for a double-edge modulator in the context of a generalised carrier. One of the disadvantages of the double-edge modulator is that the small-signal model is only valid when the duty cycle is equal to $50 \%$, as mentioned in the introduction. In contrast, the smallsignal model of the single-edge modulator is linear and time invariant for all constant values of duty cycle. This is a major advantage of the single-edge modulator.

The small-signal model described in this section can also be derived through a number of more precise, but less intuitive approaches, for instance by truncating the Taylor series of the PWM non-linearity derived in [31] or by the methods proposed in [26].

Small-signal models of regularly-sampled pulse-width modulators were derived in [32]-[34]. These small-signal models are widely used to design digital control loops with a sampling frequency that is equal to either the switching frequency or twice the switching frequency. In this case the small-signal gain is constant due to the fact that the ripple gradient is constant in a sampled system. The fact that the output signal is sampled, at fixed points within each switching period, by the analogue-to-digital converter results in a further simplification. As a result of these two facts, the small-signal models of digital control loops for regularly-sampled PWM control loops are simpler than those of continuous-time naturally-sampled PWM loops.

\section{USING THE SMALL-SIGNAL MODEL TO ANALYSE STABILITY}

Although a clear definition could not be found in the literature, the term small-signal model is usually associated with a linearisation of the underlying non-linear system around a fixed operating point [35], p54. The aim of this section is to show how the small-signal model that was derived in the previous section can be utilized to analyse the stability of a PWM feedback loop.

Fig. 4(a) shows a typical continuous-time feedback loop containing a single-edge naturally-sampled pulse-width modulator. The reference $i^{\star}(t)$ is an arbitrary signal and $\operatorname{emf}(t)$ represents a counter emf, for example as may be found in machine or grid-connected applications. In the case of a DC-to-DC converter $i^{\star}(t)$ would be a constant, while $i^{\star}(t)$ would typically be a sinusoidal reference in the case of a DC-to-AC converter. With the exception of the pulse-width modulator, all components in the loop are assumed to be linear.

Since the pulse-width modulator is non-linear, the system of differential equations that describe the loop is nonlinear. Due to the fact that the switching frequency is fixed, all the state-variables are periodic functions of time in the steady state. These state-variables form a closed phase path (also known as a trajectory or orbit) in state space, 


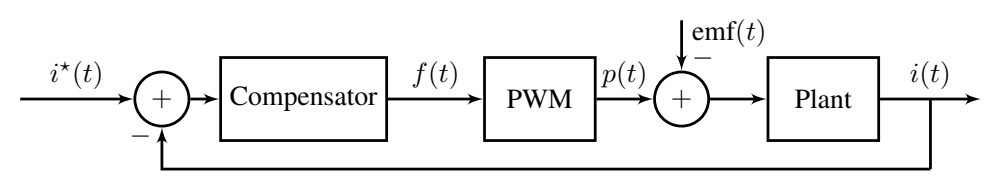

(a) PWM feedback loop with reference $i^{\star}(t)$.

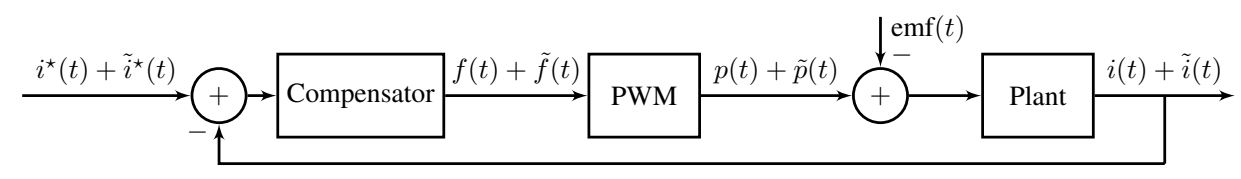

(b) PWM feedback loop with perturbed reference $i^{\star}(t)+\tilde{i}^{\star}(t)$.

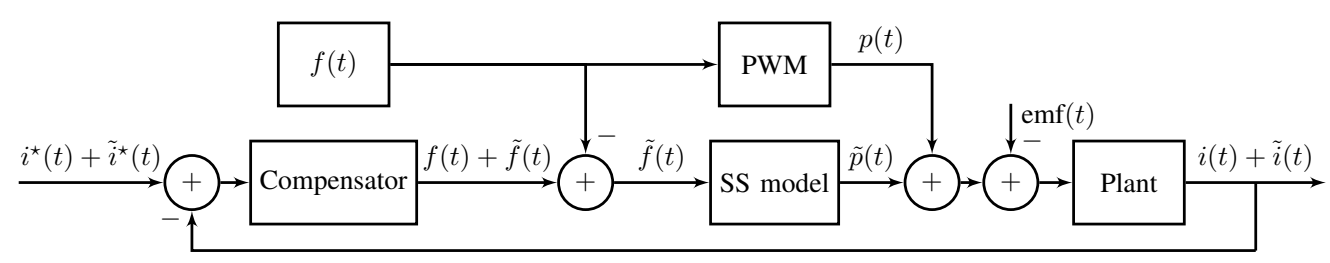

(c) Equivalent representation of the block diagram in (b).

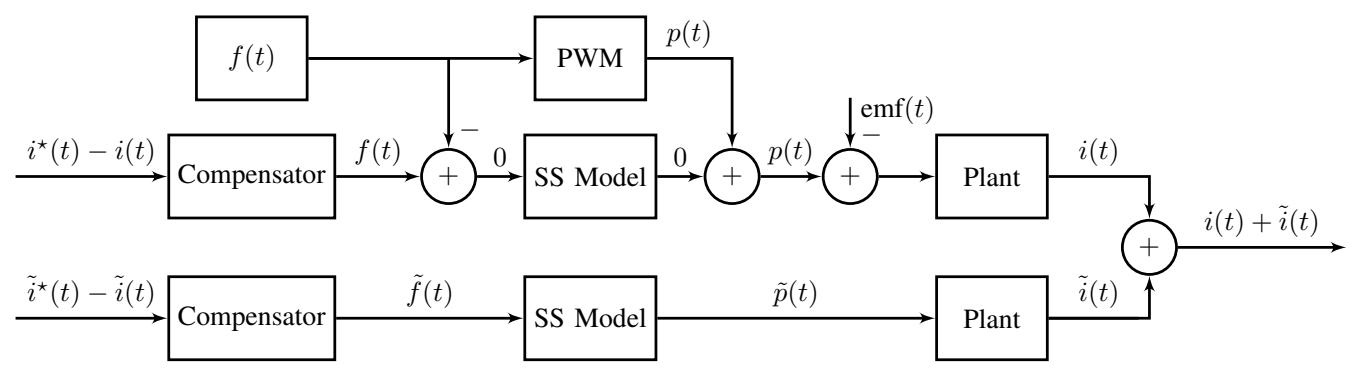

(d) By linearity, the lower branch of (c) can be split into two separate branches.

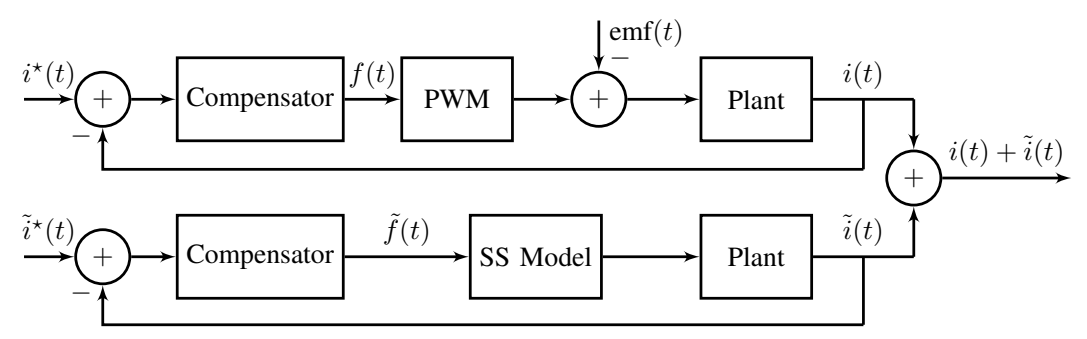

(e) The top branch of (d) is replaced by (a).

Figure 4: Splitting the loop into separate large-signal and small-signal loops. 


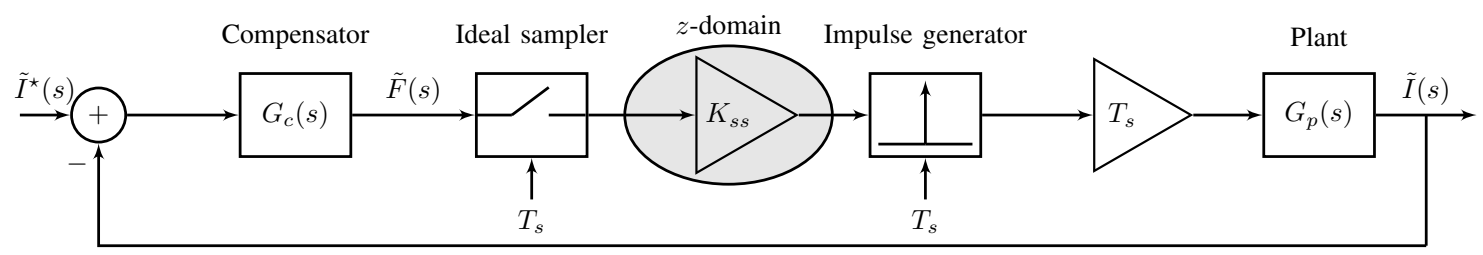

Figure 5: PWM small-signal model embedded in the feedback loop.

which is generally referred to as a limit cycle. In general, this limit cycle is not a fixed point in state-space, even when the reference $i^{\star}(t)$ is constant.

A perturbation analysis [36] is performed to analyse the input-output stability of this limit cycle. A small perturbation $\tilde{i}^{\star}(t)$ is superimposed on reference $i^{\star}(t)$ in Fig. 4(b). The question of the stability of the underlying limit cycle, is whether such a small perturbation to the reference causes corresponding small perturbations to the output signal $i(t)$ to grow over time or not. The input signal to the pulse-width modulator is now represented by $f(t)+\tilde{f}(t)$ and its output signal is $p(t)+\tilde{p}(t)$. The output signal of the control loop is $i(t)+\tilde{i}(t)$.

Fig. 4(c) shows an equivalent representation of Fig. 4(b). Since $\tilde{f}(t)$ is assumed to be a small perturbation signal, the pulse-width modulator of Fig. 4(b) can be replaced with a pulse-width modulator with input signal $f(t)$ and a PWM small-signal model with input signal $\tilde{f}(t)$.

Note that all the components in the lower branch of Fig. 4(c), i.e. the compensator, PWM small-signal model and plant are linear. As a result it is easily shown, by block diagram manipulation, that Fig. 4(d) is equivalent to Fig. 4(c). As a final step, the top branch of Fig. 4(d) can be replaced by Fig. 4(a), as shown in Fig. 4(e).

Fig. 4(e) shows how the perturbed non-linear feedback loop of Fig. 4(b) has now been separated into two parts. The top branch represents the original feedback loop of Fig. 4(a), which operates in a limit cycle. This branch will be referred to as the large-signal loop. The bottom branch is the small-signal loop and is a linear feedback loop. The location of the sampling instants as well as the small-signal gain are determined by the large-signal loop. Note that the stability of the large-signal limit cycle is reduced to analysing the stability of the linear small-signal feedback loop. It is important to note that the small-signal circuit is not a linearisation around a fixed operating point, but rather a linearisation around the large-signal limit cycle.

The small-signal model is linear and provides accurate information about the behaviour of the system in the vicinity of the original limit cycle. It can subsequently be used to analyse its stability. In general, the small-signal model does not provide other information about the large-signal behaviour of the system. Since the large-signal loop is non-linear it cannot itself be described by concepts from the theory of linear systems, like eigenvalues and transfer functions.

\section{IV. $z$-DOMAIN MODELLING OF THE SMALL-SIGNAL FEEDBACK LOOP}

Fig. 5 shows the small-signal model of Fig. 3 embedded in the lower branch of Fig 4(e). As mentioned previously, the signal passing from the ideal sampler to the impulse generator in Fig. 5 can be seen as a discrete-time signal that 


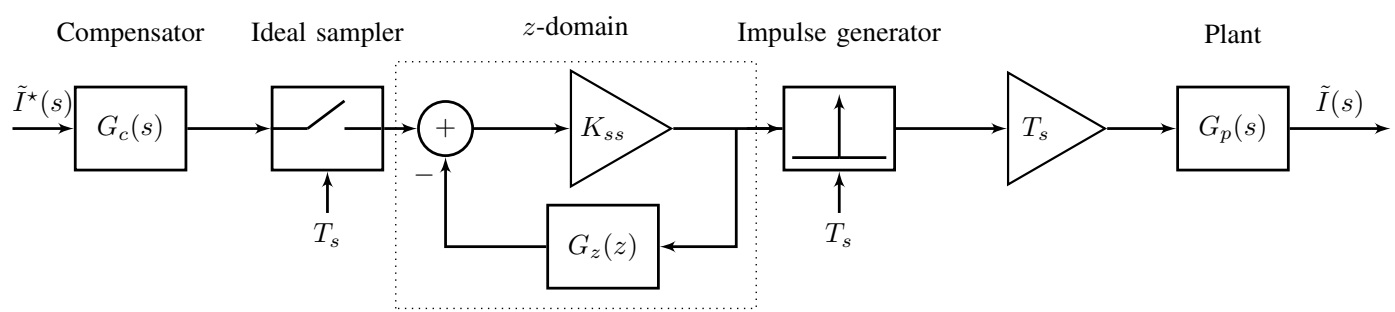

Figure 6: Equivalent block-diagram representation of Fig. 5. Note that the only feedback loop is in the $z$-domain.

describes the small-signal evolution of the duty cycle perturbation. In this section it is shown how this discrete-time signal can be studied by making use of standard $z$-domain methods.

In order to characterize the circuit from the perspective of this discrete-time domain, the link between the sampler and impulse generator in Fig. 5 is temporarily broken and the impulse generator emits a single impulse to characterize the surrounding linear circuit. The ideal sampler starts sampling at the same instant with its sampling frequency equal to the switching frequency.

Let $G(s)$ denote the open-loop $s$-domain transfer function of the surrounding continuous-time feedback loop, i.e $G(s)=G_{c}(s) G_{p}(s)$, where $G_{c}(s)$ is the compensator transfer function and $G_{p}(s)$ the plant transfer function. By expanding $G(s)$ into partial fractions [30] it can be written in the form

$$
G(s)=\sum_{k=1}^{K} \frac{A_{k}}{s+p_{k}},
$$

where the poles $-p_{1},-p_{2}, \ldots,-p_{K}$ are assumed to lie in the left-half of the complex plane. The $z$-domain transfer function $G_{z}(z)$ is obtained by sampling the impulse response $g(t)$ of $G(s)$, i.e.

$$
\begin{aligned}
G_{z}(z) & =T_{s} \sum_{n=1}^{\infty} g\left(n T_{s}\right) z^{-n} \\
& =T_{s} \sum_{k=1}^{K} A_{k}\left(\sum_{n=1}^{\infty} e^{-n p_{k} T_{s}} z^{-n}\right)
\end{aligned}
$$

Note that each of the infinite sums in Eq. (6) forms a geometric series. By using the well-known equations [37] for the sum of a geometric series, each of the infinite sums in Eq. (6) can be rewritten as

$$
\sum_{n=1}^{\infty} e^{-n p_{k} T_{s}} z^{-n}=\frac{e^{-p_{k} T s}}{z-e^{-p_{k} T_{s}}},
$$

provided that $|z|>\left|e^{-p_{k} T_{s}}\right|$. Hence

$$
G_{z}(z)=T_{s} \sum_{k=1}^{K} A_{k} \frac{e^{-p_{k} T s}}{z-e^{-p_{k} T_{s}}} .
$$

It is important to note that the first sample of the impulse response, corresponding to $n=0$, was ignored. This is because practical systems will always contain a non-zero propagation delay [17], [23]. As a result the first sample would always be zero. 
This method of transforming from the $s$-domain to the $z$-domain is a modified version of the well-known impulseinvariance method. This transformation maps the $s$-domain pole $-p_{k}$ onto the $z$-domain pole $e^{-p_{k} T_{s}}$. There is no simple way of mapping the $s$-domain zeros onto the $z$-domain zeros. The locations of the $z$-domain zeros depend on the location of the poles and their weights in the partial fraction expansion of Eq. (5). It should be noted that only $s$-domain poles in the left-half of the complex plane were considered. The theory can also be extended to poles on the imaginary axis as well as higher order poles. For the sake of simplicity it will be assumed that poles on the imaginary axis are located slightly to the left of this axis. By making a slight modification to Eq. (7) it is possible to include the effect of transport delays as was shown in [28].

Fig. 6 can be derived from Fig. 5 through simple block-diagram manipulation. Since the only feedback loop in Fig. 6 is in the $z$-domain the stability of the small-signal loop depends only on the stability of this $z$-domain loop.
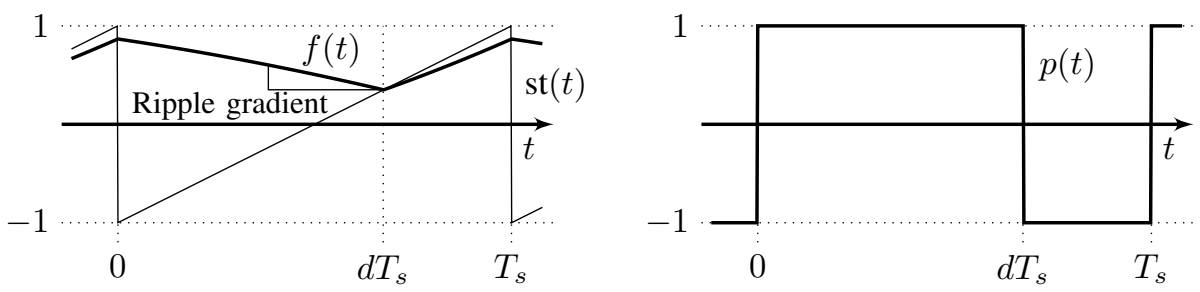

Figure 7: Typical modulator input and output signals in the steady state.

It is well-known that the impulse generator in Fig. 6 generates images around integer multiples of the switching frequency in the frequency domain. Closer investigation of Fig. 6 yields a single-frequency small-signal closed-loop transfer function:

$$
\frac{\tilde{I}(\omega)}{\tilde{I}^{\star}(\omega)}=G_{c}(j \omega)\left[\frac{K_{s s}}{1+K_{s s} G_{z}\left(e^{j \omega T_{s}}\right)}\right] T_{s} G_{p}(j \omega) .
$$

This transfer function takes into account only the harmonic at one particular frequency. It does not account for the images generated by the impulse generator or the PWM sidebands generated by the pulse-width modulator. It is similar to the transfer characteristic measured by a narrow-band gain-phase analyser. A similar transfer function was derived in [24], [25] within the context of a generalised carrier.

\section{AN ANALYTIC EQUATION FOR THE SMALL-SIGNAL GAIN}

The PWM pulse train contains high-frequency ripple components that are injected back into the feedback loop. Fig. 7 shows a typical modulator input signal $f(t)$ as well as its output signal $p(t)$ in the steady state. The duty cycle is denoted by $d$. According to Eq. (4), the small-signal gain $K_{s s}$ of the pulse-width modulator depends on the gradient of the input signal to the pulse-width modulator just prior to its intersection with the carrier. The small-signal gain would be equal to 1 in the absence of ripple feedback. In most cases, ripple feedback causes a reduction in the small-signal gain.

One solution to the problem of ripple feedback would be to place a low-pass filter in the feedback path to reduce the PWM ripple before entering the pulse-width modulator. However, the additional delay caused by this filter 


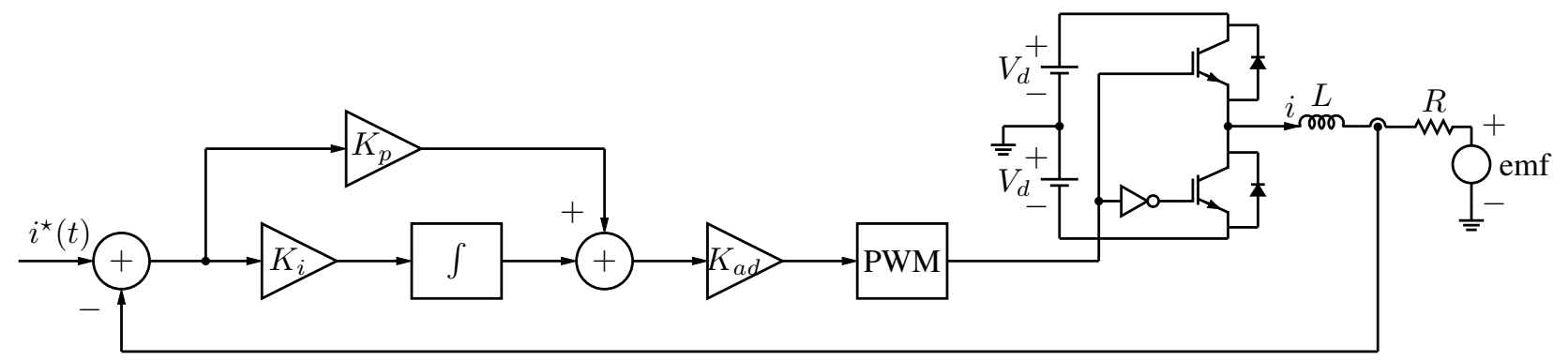

Figure 8: Circuit used for the design example.

imposes unnecessary restrictions in terms of gain and bandwidth on the design of the regulator. Developing a clear understanding of the effect of ripple feedback makes it possible to design high-performance feedback loops without any unnecessary low-pass filtering.

To this end, a closed-form solution for the steady-state value of the ripple gradient was derived. The mathematical details of this derivation can be found in the appendix. The gradient $f^{\prime}\left(t_{s p}\right)$ of the modulator input signal $f(t)$ just prior to its intersection with the carrier is given by:

$$
f^{\prime}\left(t_{s p}\right)=\lim _{\substack{t \rightarrow d T_{s} \\ t \leq d T_{s}}}\left[\frac{d f(t)}{d t}\right]=2 \sum_{k=1}^{K} A_{k}\left(\frac{e^{-p_{k} T_{s}}-e^{-p_{k} d T_{s}}}{1-e^{-p_{k} T_{s}}}\right) .
$$

According to Eq. (4), the small-signal gain $K_{s s}$ of the pulse-width modulator is given by:

$$
K_{s s}=\frac{f_{s}}{f_{s}-\sum_{k=1}^{K} A_{k}\left(\frac{e^{-p_{k} T_{s}}-e^{-p_{k} d T_{s}}}{1-e^{-p_{k} T_{s}}}\right)} .
$$

As expected, the small-signal gain depends on the duty cycle of the pulse-width modulator and is equal to 1 when the duty cycle equals 1.

Remark: During the derivation of Eq. (9) it was assumed that all the poles of $G(s)$ are located in the left half of the complex plane. If $p_{k}$ is located at the origin, i.e. $p_{k}=0$, then the contribution of $p_{k}$ to the sum in Eq. (9) can be calculated by taking the limit as $p_{k} \rightarrow 0$, i.e.

$$
\lim _{p_{k} \rightarrow 0} A_{k}\left(\frac{e^{-p_{k} T_{s}}-e^{-p_{k} d T_{s}}}{1-e^{-p_{k} T_{s}}}\right)=A_{k}(d-1) .
$$

\section{VI. $z$-DOMAIN DESIGN STRATEGY}

A major advantage of the small-signal model and the resulting $z$-domain analysis is that it gives rise to an accurate but simple design strategy. As an example, the design of a PI current regulator with an $R L$ load as shown in Fig. 8 is outlined in this section. The gain block $K_{a d}$ in Fig. 8 represents an additional gain term which will be used in subsequent sections. It is assumed that $K_{a d}=1$ throughout this section. The system parameters are shown in Table I. 
Table I: System parameters

\begin{tabular}{|l|l|l|}
\hline Parameter & Symbol & Value \\
\hline \hline Inductor & $L$ & $17 \mathrm{mH}$ \\
Resistor & $R$ & $10 \Omega$ \\
Bus voltage & $V_{d}$ & $200 \mathrm{~V}$ \\
Switching frequency & $f_{s}$ & $5 \mathrm{kHz}$ \\
Counter EMF & $\mathrm{emf}$ & $30 \mathrm{~V}$ \\
\hline
\end{tabular}

Assume for the moment that the small-signal gain $K_{s s}$ is equal to 1 and let $\tau:=\frac{L}{R}$ denote the time-constant of the $R L$ load. The open-loop transfer function $G(s)$ for the system of Fig. 8 is given by

$$
G(s)=G_{c}(s) G_{p}(s)=\left(K_{p}+\frac{K_{i}}{s}\right)\left(\frac{\frac{V_{d}}{L}}{s+\frac{1}{\tau}}\right) .
$$

The first step is to expand $G(s)$ into partial fractions according to Eq (5), i.e.

$$
G(s)=\frac{A_{1}}{s+p_{1}}+\frac{A_{2}}{s+p_{2}}
$$

where

$$
p_{1}=0, \quad p_{2}=\frac{1}{\tau}, \quad A_{1}=\frac{K_{i} V_{d}}{R} \text { and } A_{2}=V_{d}\left(\frac{K_{p}}{L}-\frac{K_{i}}{R}\right) .
$$

The next step is to transform the $s$-domain transfer function $G(s)$ to the $z$-domain by making use of the impulse invariance method described by Eq. (7), i.e.

$$
\begin{aligned}
G_{z}(z) & =V_{d}\left[\frac{K_{i}}{R} \frac{1}{z-1}+\left(\frac{K_{p}}{L}-\frac{K_{i}}{R}\right) \frac{e^{-\frac{T_{s}}{\tau}}}{z-e^{-\frac{T_{s}}{\tau}}}\right] T_{s} \\
& =\left[\frac{\frac{V_{d}}{L}}{z-e^{-\frac{T_{s}}{\tau}}}\right]\left[K_{p} e^{-\frac{T_{s}}{\tau}}+\frac{K_{i} \tau\left(1-e^{-\frac{T_{s}}{\tau}}\right) z}{z-1}\right] T_{s} .
\end{aligned}
$$

The first term:

$$
\left[\frac{\frac{V_{d}}{L}}{z-e^{-\frac{T_{s}}{\tau}}}\right]
$$

of $G_{z}(z)$ represents the $R L$ load. The $s$-domain pole located at $-\frac{1}{\tau}$ is mapped onto the $z$-domain pole at $e^{-\frac{T_{s}}{\tau}}$. It is quite interesting that the second term:

$$
\left[K_{p} e^{-\frac{T_{s}}{\tau}}+\frac{K_{i} \tau\left(1-e^{-\frac{T_{s}}{\tau}}\right) z}{z-1}\right]
$$

corresponds to the transfer function of a discrete-time PI controller [34] whose transfer function is of the form:

$$
G_{P I}(z)=K_{p}^{\prime}+K_{i}^{\prime}\left(\frac{z}{z-1}\right)=K_{p}^{\prime}+\frac{K_{i}^{\prime}}{1-z^{-1}},
$$

where

$$
K_{p}^{\prime}=K_{p} e^{-\frac{T_{s}}{\tau}} \quad \text { and } \quad K_{i}^{\prime}=K_{i} \tau\left(1-e^{-\frac{T_{s}}{\tau}}\right)
$$


Note that the two coefficients, $K_{p}^{\prime}$ and $K_{i}^{\prime}$, of the discrete-time PI controller are just scaled versions of the coefficients $K_{p}$ and $K_{i}$ of the original continuous-time PI controller $G_{c}(s)$.

A variety of design techniques can be utilised to design this discrete-time PI controller in the $z$-domain. Once the values of $K_{p}^{\prime}$ and $K_{i}^{\prime}$ are determined, the values of $K_{p}$ and $K_{i}$ can then be calculated from Eq. (14), yielding the continuous-time controller.

For this example it was decided to design for a crossover frequency $\omega_{c}$ of $2 \pi \times 1000 \mathrm{rad} / \mathrm{s}$ and a phase margin $P M$ of $45^{\circ}$ in the $z$ domain. It is well known that the frequency response of the $z$-domain transfer function $G_{z}(z)$ is obtained by evaluating $G_{z}(z)$ on the unit circle by setting $z=e^{j \omega T_{s}}$ [38]. By definition, the magnitude of open-loop transfer function $G_{z}\left(e^{j \omega T_{s}}\right)$ must be equal to 1 and its phase must be equal to $-\left(180^{\circ}-P M\right)$ at the crossover frequency $\omega=\omega_{c}$, i.e.

$$
G_{z}\left(e^{j \omega_{c} T_{s}}\right)=e^{j \frac{(P M-180) \pi}{180}} .
$$

According to equations (12) and (13), this implies that

$$
e^{j \frac{(P M-180) \pi}{180}}=T_{s} \frac{V_{d}}{L\left(e^{j \omega_{c} T_{s}}-e^{-\frac{T_{s}}{\tau}}\right)}\left(K_{p}^{\prime}+\frac{K_{i}^{\prime}}{1-e^{-j \omega_{c} T_{s}}}\right) .
$$

Hence

$$
\frac{L}{T_{s} V_{d}}\left(e^{j \omega_{c} T_{s}}-e^{-\frac{T_{s}}{\tau}}\right) e^{j \frac{(P M-180) \pi}{180}}=\left(K_{p}^{\prime}+\frac{K_{i}^{\prime}}{1-e^{-j \omega_{c} T_{s}}}\right) .
$$

By comparing the real and imaginary parts of the two sides of this equation, the following two expressions for $K_{i}^{\prime}$ and $K_{p}^{\prime}$ are derived:

$$
K_{i}^{\prime}=\frac{\Im\left(\gamma_{1}\right)}{\Im\left(\gamma_{2}\right)} \quad \text { and } \quad K_{p}^{\prime}=\Re\left(\gamma_{1}-K_{i}^{\prime} \gamma_{2}\right)
$$

where $\gamma_{1}$ and $\gamma_{2}$ are defined as

$$
\gamma_{1}=\frac{L}{T_{s} V_{d}}\left(e^{j \omega_{c} T_{s}}-e^{-\frac{T_{s}}{\tau}}\right) e^{j \frac{\left(P M-180^{\circ}\right) \pi}{180^{\circ}}} \quad \text { and } \quad \gamma_{2}=\frac{1}{1-e^{-j \omega_{c} T_{s}}} .
$$

Note that $\Re$ and $\Im$ denote the real and imaginary parts of a complex number, respectively.

Calculating the values of $K_{i}^{\prime}$ and $K_{p}^{\prime}$ from Eq. (15) yielded $K_{i}^{\prime}=0.1620 \mathrm{rad} / \mathrm{s}$ and $K_{p}^{\prime}=0.3791$. The parameters $K_{p}$ and $K_{i}$ of the continuous-time PI controller were subsequently calculated from Eq. (14), which yielded $K_{p}=$ 0.4264 and $K_{i}=858.7758 \mathrm{rad} / \mathrm{s}$.

The next step is to take the dependence of the small-signal gain $K_{s s}$ on the duty cycle $d$ into consideration. Substituting the coefficients of Eq. (11) into Eq. (10), results in the following expression for the small-signal gain:

$$
K_{s s}=\frac{f_{s}}{f_{s}-V_{d}\left[\frac{K_{i}}{R}(d-1)+\left(\frac{K_{p}}{L}-\frac{K_{i}}{R}\right)\left(\frac{e^{-\frac{T_{s}}{\tau}}-e^{-\frac{d T_{s}}{\tau}}}{1-e^{-\frac{T_{s}}{\tau}}}\right)\right]} .
$$

This equation was used to plot Fig. 9, which shows the small-signal gain as a function of the duty cycle. The small-signal gain varies between 0.5 and 1 , with $K_{s s}$ reaching 1 at a duty cycle of 1 . This shows that the overall loop gain is reduced as a result of ripple feedback. Fig. 10 shows the open-loop Bode plot of $G_{z}(z)$ for the cases $K_{s s}=1$ and $K_{s s}=0.5$. The value of the phase-margin increases from $45^{\circ}$ to $54^{\circ}$ when the small-signal gain 


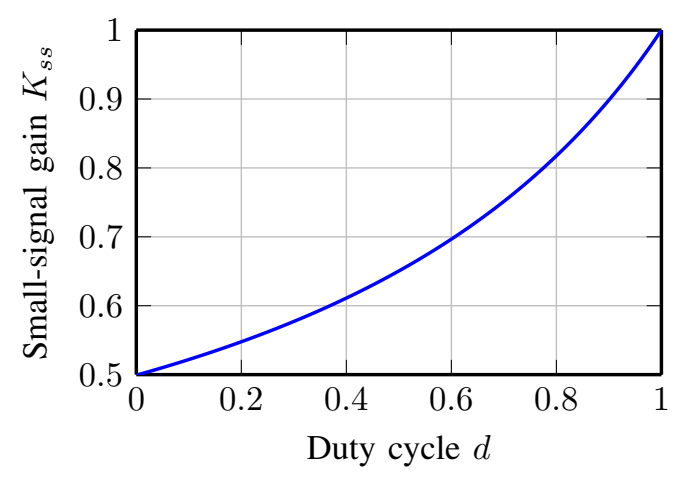

Figure 9: Small-signal gain as a function of duty cycle.

decreases from 1 to 0.5 . At the same time the gain margin increases from $4.8 \mathrm{~dB}$ to $10.8 \mathrm{~dB}$. This shows that the relative stability of the loop improves as $K_{s s}$ is reduced. This justifies the assumption, made at the beginning of this section, that $K_{s s}=1$, since it results in a worst-case design. One disadvantage of the dependence of $K_{s s}$ on the duty cycle is that fact that the crossover frequency is reduced from $1000 \mathrm{~Hz}$ when $K_{s s}=1$ to $500 \mathrm{~Hz}$ when $K_{s s}=0.5$. This has the effect that the small-signal bandwidth of the loop is reduced when the duty cycle is small. A ripple compensation strategy, which greatly reduces the dependence of the ripple gradient on value of the duty cycle, is described in [28], [39], [40].

Since the small-signal gain $K_{s s}$ depends on the duty cycle, the locations of the closed-loop poles become dependent on the duty cycle as shown in the $z$-domain root locus of Fig. 11.

For more complex designs, a number of Matlab $\AA$ functions and tools can be utilised during the design procedure. The residue function can be used to calculate the partial fraction expansion, while the Control System Designer can be used for the $z$-domain design.

A less precise design strategy was used to design a PR controller in [28], where the $z$-domain design was carried out by placing the open-loop zeroes and adjusting the gain by hand. Furthermore, the design process was not discussed in detail in [28] and the ripple gradient (and hence the small-signal gain) had to be determined by means of time-domain simulations. In [40] a state-of-the-art class-d amplifier with a fifth-order voltage control loop was designed by making use of a more sophisticated numerical design optimisation strategy. An experimental evaluation of this amplifier can be found in [40], [41].

\section{MODEL VERIFICATION}

This section presents a number of time-domain simulations of the controller designed in the previous section, to verify the validity of the small-signal model and the resulting $z$-domain analysis. In the process the mechanisms that lead to instability are clarified, the concept of the critical loop gain is introduced and its closed-form mathematical expression is derived. 

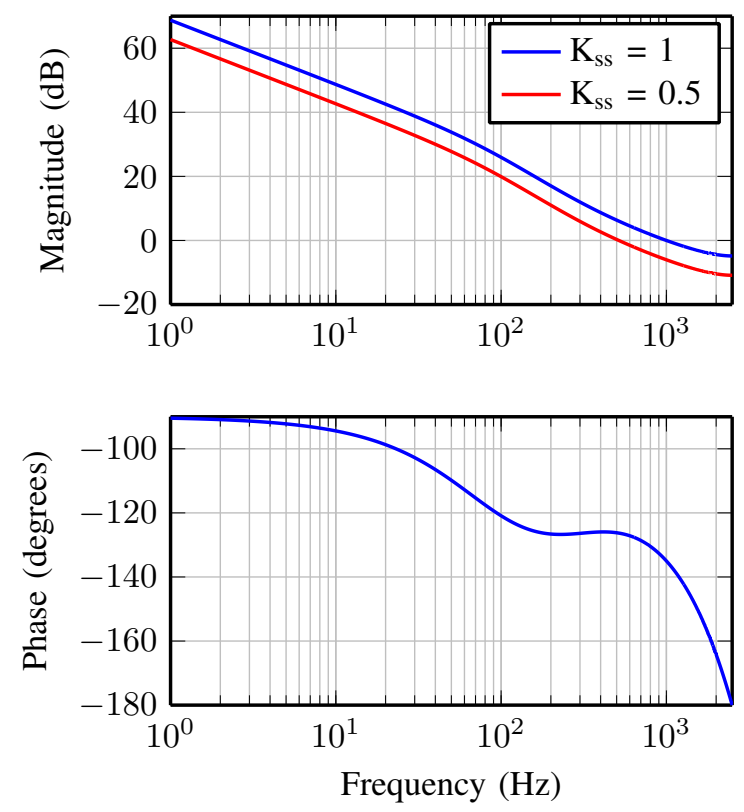

Figure 10: Open-loop $z$-domain bode plot.

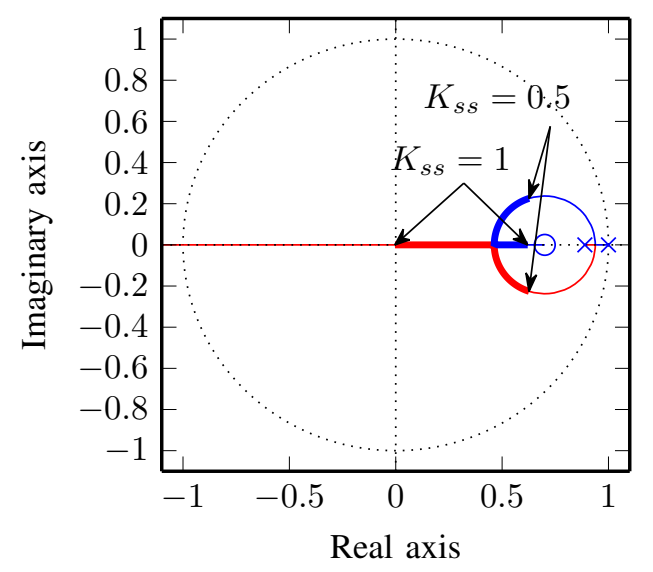

Figure 11: $z$-domain root locus.

\section{A. Gain margin}

In order to verify the gain margin predicted by the small-signal model, the value of the additional gain term $K_{a d}$ in Fig. 8 is slowly ramped up and a bifurcation diagram is constructed to identify the value of $K_{a d}$ at which instability occurs. When ramping up the value of $K_{a d}$ the ripple gradient increases accordingly, thereby reducing the small-signal gain. The value $K_{c r i t}$ of $K_{a d}$ at which instability occurs is given by $G_{m}=K_{a d} K_{s s}$, where $G_{m}$ 


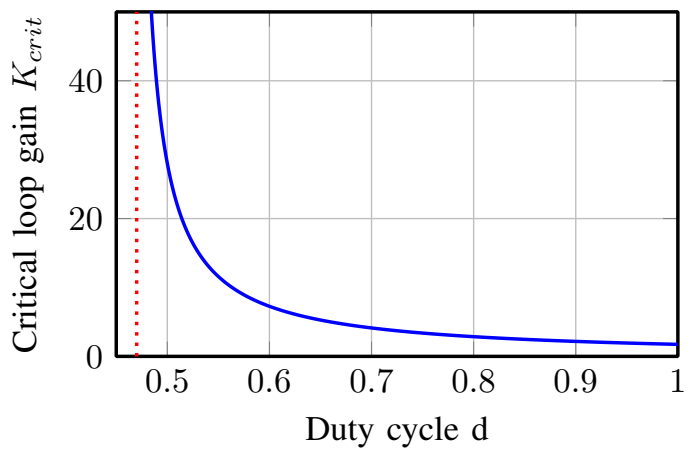

Figure 12: Critical gain $K_{c r i t}$ as a function of duty cycle. The red asymptote is located at $d=0.47$.

is the gain margin of $G_{z}(z)$. According to Eq. (10) this occurs when:

$$
G_{m}=\frac{K_{a d} f_{s}}{f_{s}-K_{a d} \sum_{k=1}^{K} A_{k}\left(\frac{e^{-p_{k} T_{s}}-e^{-p_{k} d T_{s}}}{1-e^{-p_{k} T_{s}}}\right)} .
$$

Note that the term $K_{a d}$ in the denominator accounts for the fact that the ripple gradient also increases by a factor $K_{a d}$. Solving for $K_{\text {crit }}$ yields:

$$
K_{\text {crit }}=\frac{G_{m} f_{s}}{f_{s}+G_{m} \sum_{k=1}^{K} A_{k}\left(\frac{e^{-p_{k} T_{s}}-e^{-p_{k} d T_{s}}}{1-e^{-p_{k} T_{s}}}\right)} .
$$

Fig. 12 shows a graph of $K_{\text {crit }}$ as a function of the duty cycle. For values of $d<0.46$ it is impossible to make the loop go unstable by simply increasing $K_{a d}$. Increasing $K_{a d}$ increases the ripple gradient. This results in a decrease in the small-signal gain $K_{s s}$ which decreases more rapidly than $K_{a d}$ increases, resulting in an overall decrease in gain in the region $d<0.46$.

At a duty cycle of $d=1$ the critical loop gain equals the gain margin of $G_{z}(z)$. This is due to the fact that the small-signal gain $K_{s s}$ equals 1 when $d=1$.

The value of $K_{a d}$ was gradually ramped up over a period of $2000 \mathrm{~s}$ to generate the bifurcation diagrams of Fig. 13, in which the x-axis represents the value of $K_{a d}$. Such bifurcation diagrams are a valuable tool to analyse the stability of the underlying limit cycle. Since $K_{a d}$ was ramped up linearly in time, the $x$-axis is a scaled version of the time axis. Each dot on the bifurcation diagram represents a value of the duty cycle. The duty cycle remains constant up to the bifurcation point, after which it starts to oscillate, indicating the onset of instability.

The dotted lines in these graphs indicate the critical value $K_{\text {crit }}$ of $K_{a d}$ according to the theory. The current reference in Fig. 13(a) was set to $-10 \mathrm{~A}$, which results in a duty cycle of 0.335 . This value is less than 0.46 and hence, according to Fig. 12, the loop cannot be driven into unstable operation by increasing the value of $K_{a d}$. The simulated and theoretical bifurcation points in the other three graphs of Fig. 13 agree very well. In each case, the duty cycle starts to oscillate at half the switching frequency when the loop gain reaches the theoretical stability 

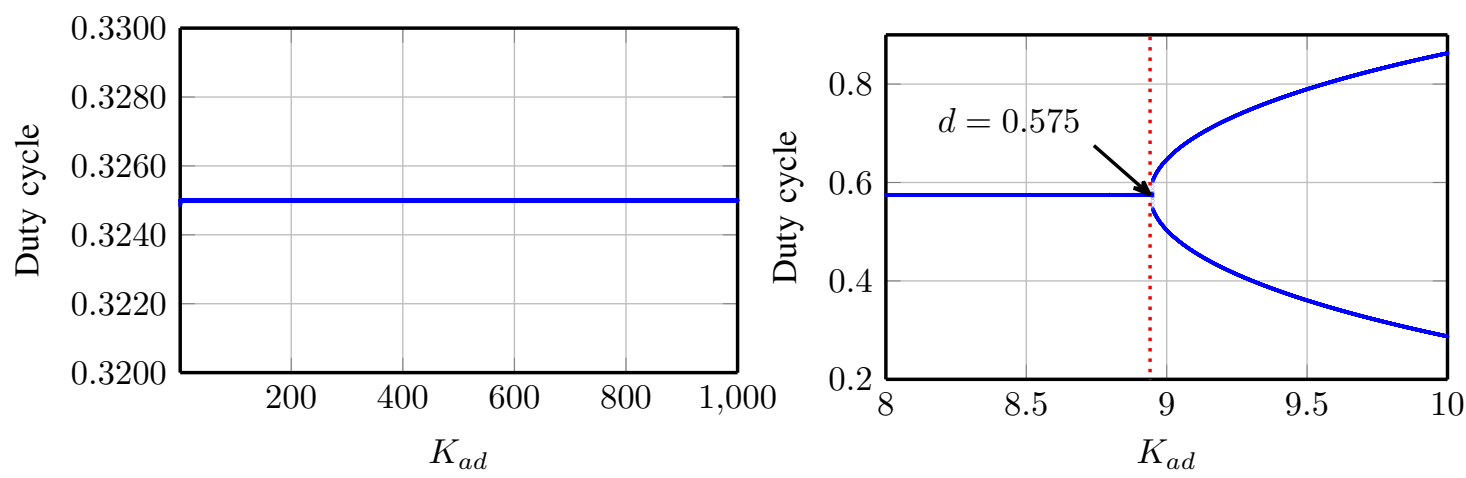

(a) Bifurcation diagram with $I_{r e f}=-10$ A.

(b) Bifurcation diagram with $I_{r e f}=0$ A.
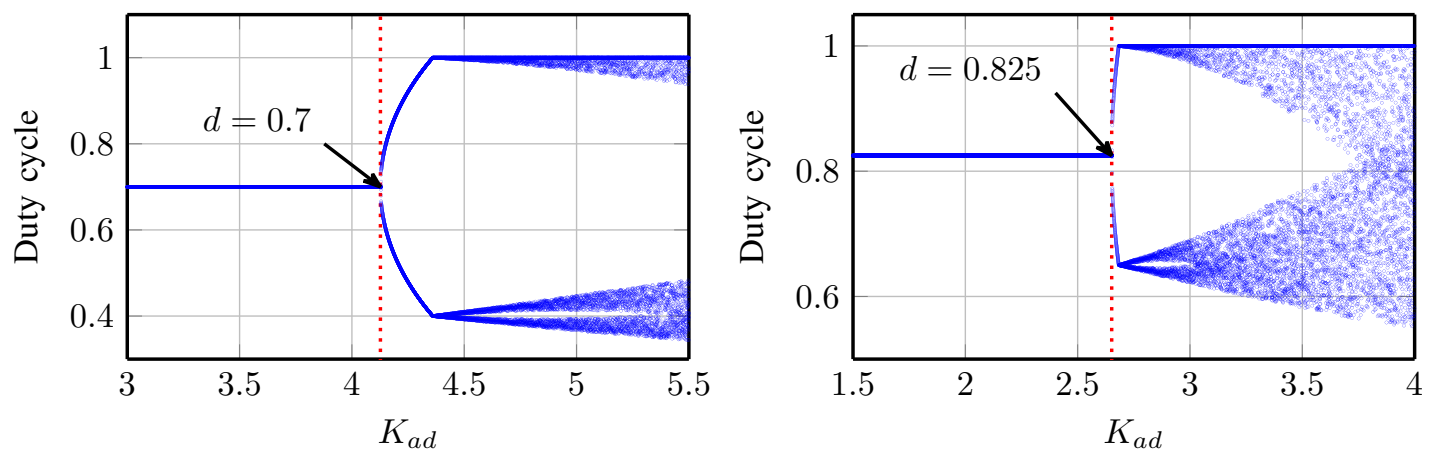

(c) Bifurcation diagram with $I_{r e f}=5$ A.

(d) Bifurcation diagram with $I_{r e f}=10$ A.

Figure 13: Bifurcation diagrams. The dotted lines show the theoretical value of the critical loop gain.

boundary. The bifurcation is thus a period-doubling bifurcation, consistent with the fact that the $z$-domain root locus exits the unit circle at -1 in Fig. 11.

In a linear system, instability implies that at least one of the state variables will go to $\pm \infty$. In contrast, after the onset of instability at the bifurcation point in this non-linear system, the loop simply changes its mode of operation, indicating that the bifurcation is supercritical. Another bifurcation occurs when $K_{a d}$ is increased to the point where the pulse-width modulator is driven into saturation. The small-signal model presented in this paper only predicts the first bifurcation point. However, as can be seen for the bifurcation diagram with $I_{r e f}=10 \mathrm{~A}$, the second bifurcation can occur soon after the first bifurcation, when the converter operates at a duty cycle close to 1 . As a result the period-doubling mode of operation is seen as an unwanted mode.

It is important to point out that the traditional average model of the pulse-width modulator predicts an infinite gain margin, irrespective of the value of the duty cycle. It is thus evident that the average model does not provide an accurate indication of the stability margins of this simple control loop. This instability can be attributed to the sampling behaviour of the pulse-width modulator. 

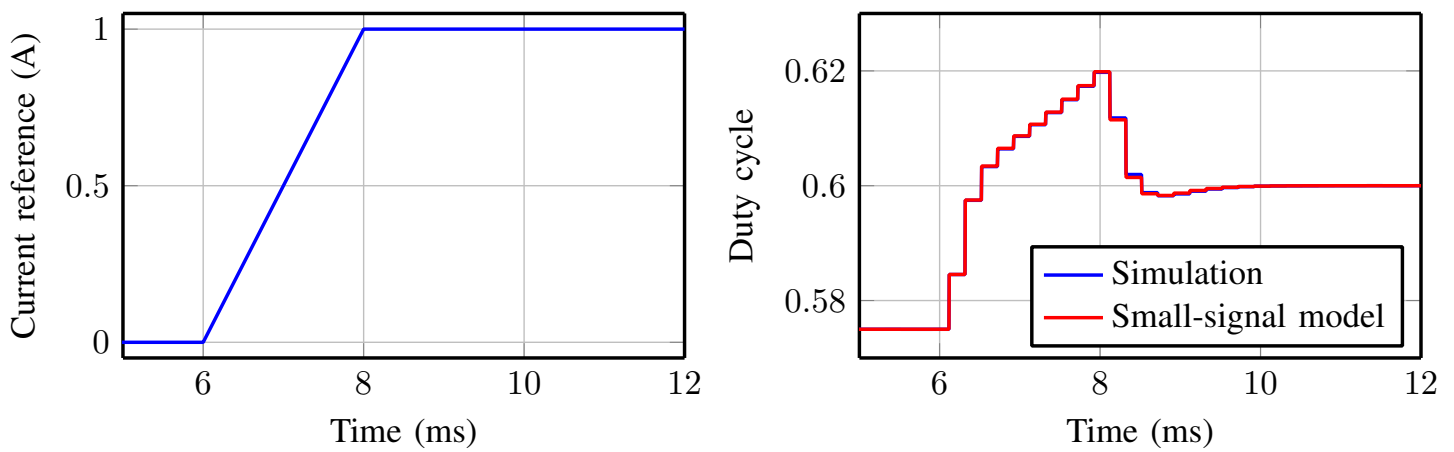

(a) Ramp in current reference.

(b) Large-signal and small-signal ramp response.

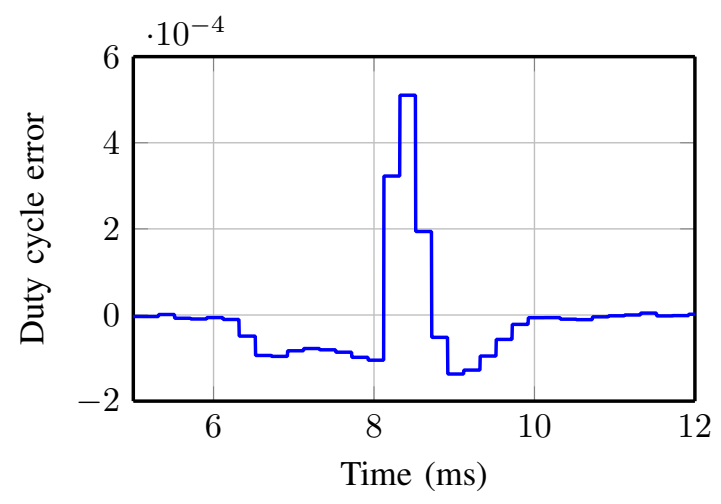

(c) Difference between the two curves in (b). Note that the maximum error is only $0.5 \%$.

Figure 14: Ramp response.

\section{B. Small-signal transient response}

Another way to validate the small-signal model is by simulating the transient response of the current regulator. A simulation of the converter was carried out in which the current reference $i^{\star}(t)$ was initially set to $0 \mathrm{~A}$. The current reference was then ramped up from 0 to 1 A over a period of $2 \mathrm{~ms}$ as shown in Fig. 14(a). The $z$-domain feedback loop of Fig. 6 was also simulated to obtain the small-signal transient response. The results of this simulation are shown in Fig. 14(b), while Fig. 14(c) shows the duty cycle error (difference between the two curves in (b)). The transient response predicted by the small-signal model corresponds closely to that of the simulation, with the maximum difference between the two being equal to approximately $5.3 \times 10^{-4}=0.053 \%$. Perfect agreement should not be expected since the small-signal model is a linearisation of the non-linear system.

\section{Small-signal closed-loop transfer function}

The small-signal closed-loop transfer function predicted by Eq. (8) is verified in this subsection. In order to measure this transfer function through simulation, a sinusoidal signal $i^{\star}(t)=I \sin (\omega t)$ is used as the current 

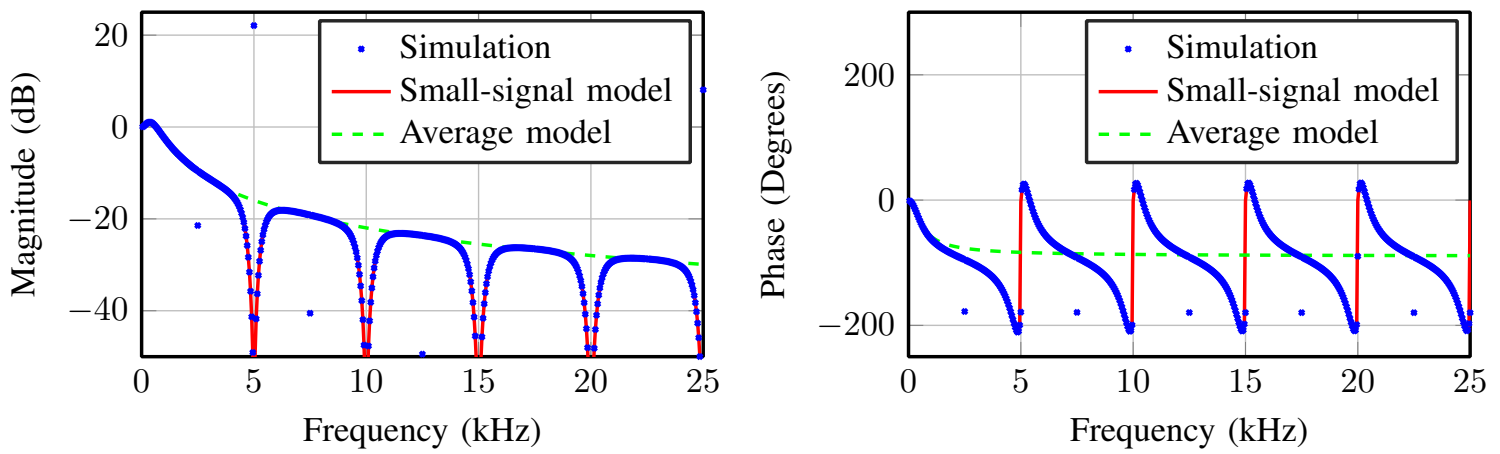

Figure 15: Small-signal closed-loop frequency response. The magnitude response is shown on the left and the phase response on the right.

reference for the circuit of Fig. 8 and the feedback loop is simulated in the time domain. The results from the first $20 \mathrm{~ms}$ of the simulation are discarded since they contain the start-up transient response of the loop. The remaining time-domain results are used to calculate the magnitude and phase of the harmonic at frequency $\omega$ of the output current $i(t)$. The magnitude of this harmonic is divided by the magnitude $I$ of the reference $i^{\star}(t)$. This produces a dot on the magnitude plot of Fig. 15. The phase of the harmonic at $\omega$ of the output current $i$ produces a dot at $\omega$ on the phase plot. The value of $\omega$ is increased and the process is repeated.

It is important to keep in mind that the impedance of the $R L$ load increases with increasing frequency. Hence the amplitude $I$ of the reference $i^{\star}(t)$ must be decreased accordingly. The results of Fig. 15 were obtained with a maximum duty cycle deviation of $10 \%$.

Fig. 15 also shows the magnitude and phase response of the small-signal transfer function according to Eq. (8) as well as the transfer function predicted by the average model of the pulse-width modulator. It is evident that, with the exception of the points at integer multiples of half the switching frequency, the results from Eq. (8) agree very well with those of the time-domain simulation. The full PWM spectrum contains images of the reference signal at multiples of half the switching frequency. These spectral components were not taken into account in the derivation of Eq. (8). The full PWM spectrum also contains PWM sidebands around multiples of the switching frequency. These sidebands are also not predicted by the small-signal model.

Fig. 15 shows that the widely-used average model of the pulse-width modulator is accurate up to approximately half the switching frequency. It provides an accurate prediction of the magnitude response, except near multiples of the switching frequency, where it is very inaccurate. It is clear that the average model does not provide an accurate prediction of the phase response above half the switching frequency. This is again due to the fact that the average model does not take the sampling behaviour of the pulse-width modulator into account. 

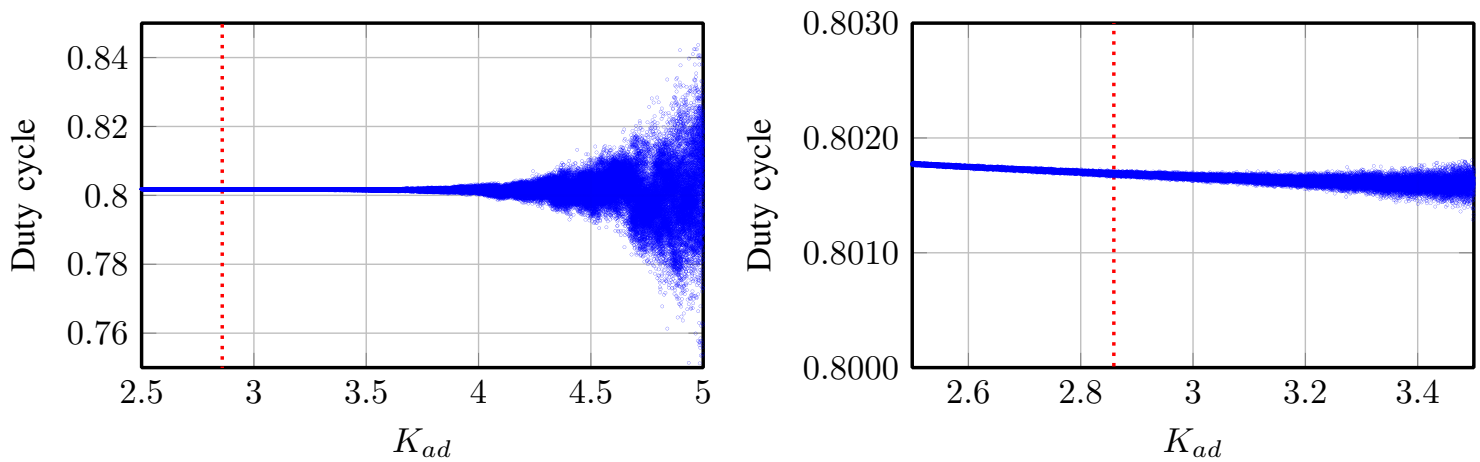

Figure 16: Bifurcation diagram with a sinusoidal reference current. The dotted line indicates the critical loop gain. The figure on the right is a zoomed view near the bifurcation point.

\section{Sinusoidal reference}

In this subsection it is shown how the small-signal model can be used to analyse the stability of the current regulator of Fig. 8 for a sinusoidal current reference $i^{\star}(t)$.

In order to apply the $z$-domain analysis strategy, it is assumed that the feedback loop is always in a quasi-static state. This is an accurate assumption, provided the the reference current $i^{\star}(t)$ varies slowly in comparison with the time constants of the rest of the system.

The system of Fig. 8 was simulated with a $50 \mathrm{~Hz}$ sinusoidal reference with an amplitude of 8 A. Presenting the effects of instability on a sinusoidal reference is particularly challenging. The small-signal gain varies over a $50 \mathrm{~Hz}$ cycle and reaches its maximum value when the duty cycle reaches its maximum value. As the loop gain is increased beyond the bifurcation point the system goes into unstable operation for very short periods of time, around the maximum value of the duty cycle. As the duty cycle decreases, the small-signal gain starts to decrease and the loop goes back into stable operation. This makes it hard to detect the bifurcation point, since the disturbances tend to grow slowly in time. The strategy that was used in this subsection was to sample the duty cycle once per fundamental $(20 \mathrm{~ms})$ period, just after the maximum value of the duty cycle is reached. Fig. 16 shows the resulting bifurcation diagram.

The zoomed view in Fig. 16 shows that the disturbance in the duty cycle starts to oscillate just before the critical value of loop gain of 2.859 is reached, confirming the theoretical prediction of the bifurcation point. This shows that the theory remains accurate under sufficiently slow sinusoidal modulation. The presence of stable operation over the majority of the $50 \mathrm{~Hz}$ cycle has the consequence that disturbances are strongly damped and hence the duty-cycle disturbances, evident in Fig. 16 beyond the instability threshold, are small. Results on the delay when observing bifurcations where a parameter is slowly varied can be found in the mathematical literature [42].

Fig. 17 shows the duty cycle over one fundamental period. The additional loop gain $K_{a d}$ was kept constant at 4.25 for this simulation. According to Fig. 12, instability is expected when the duty cycle reaches 0.802 . The duty cycle reaches 0.802 at approximately $7 \mathrm{~ms}$ and the first oscillations in the duty cycle can be observed slightly 


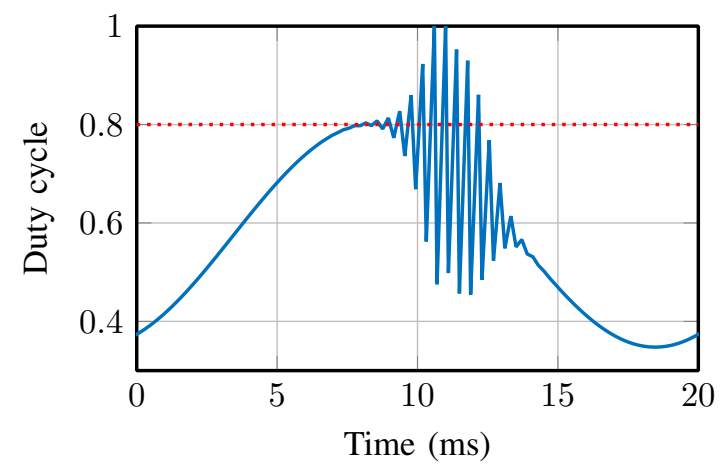

Figure 17: Duty cycle $d$ over one $20 \mathrm{~ms}$ cycle with a sinusoidal reference and $K_{a d}=4.25$. The dotted red line shows the theoretical value of $d$ at which instability is expected.

before $7 \mathrm{~ms}$, indicating instability. At approximately $13 \mathrm{~ms}$ the duty cycle drops below the critical value of 0.802 . The oscillations in the duty cycle disappear shortly after, indicating a return to stable operation.

\section{CONCLUSIONS AND FUTURE WORK}

This paper showed how the behaviour of naturally-sampled single-edge PWM control loops can easily be analysed by using methods from classical control theory. A small-signal model, according to which the pulse-width modulator consists of an ideal sampler, a small-signal gain and an impulse generator, was derived. Also, an analytic equation for the duty-cycle dependence of the small-signal gain, which depends on the ripple gradient, was derived. The effect of the ripple gradient was explicitly incorporated into the small-signal model. The sampling behaviour of the pulse-width modulator gives rise to a discrete-time signal of which the stability can be analysed by using standard $z$-domain methods. A design strategy, according which the continuous-time controller is designed in the $z$-domain, was developed.

The validity of the approach presented in this paper was verified by means of detailed comparisons with timedomain simulations. The mechanisms that lead to instability were analysed in detail and an expression for the critical loop gain was derived. The strategy that was presented in this paper does not require the use of timedomain simulations to find the steady-state operating point, nor to calculate the ripple gradient.

The small-signal model can be generalised to the case of the double-edge modulator. In this case the PWM input signal is sampled twice per switching period, with unequal sampling periods. Dealing with the unequal sampling periods presents a more complicated problem which can be solved by the introduction of dummy state variables. This will be the topic of a follow-up paper.

\section{APPENDIX}

\section{DERIVATION OF AN EQUATION FOR THE RIPPLE GRADIENT}

It is assumed that the reference signal $i^{\star}(t)$ of the control loop of Fig. 4(a) is constant and the system is assumed to be in its steady state. The ripple component of $f(t)$ is the response of the open-loop transfer function $G(s)$ 
to the modulator output waveform $p(t)$. Since the reference $i^{\star}(t)$ is assumed to be constant it only influences the DC-component of $f(t)$ and does not influence the gradient of $f(t)$. Hence the DC-component of $f(t)$ is not taken into account in this analysis.

In order to derive an analytic equation for the ripple gradient, the open-loop transfer function $G(s)$ is again decomposed into partial fractions as in Eq. (5). Each of the terms in this partial fraction expansion can be represented by the simple feedback loop shown in Fig. 18. The output of the $k^{\prime}$ th integrator in this expansion is denoted by $x_{k}(t)$. Note that the $A C$-component $f_{A C}(t)$ of the modulator input signal $f(t)$ is given by

$$
f_{A C}(t)=\sum_{k=1}^{K} A_{k} x_{k}(t)
$$

The response of each of the simple feedback loops in Fig. 18 to the pulse-width modulated block wave $p(t)$ is now analysed individually.

Let $x_{k}(0)$ denote the value of $x_{k}$ at the start of the switching period. By applying duHamel's formula [43] over the interval $0 \leq t \leq d T_{s}$, it is possible to calculate $x_{k}\left(d T_{s}\right)$ :

$$
\begin{aligned}
x_{k}\left(d T_{s}\right) & =e^{-p_{k} d T_{s}} x_{k}(0)-\int_{0}^{d T_{s}} e^{-(t-\xi) p_{k}} d \xi \\
& =e^{-p_{k} d T_{s}} x_{k}(0)-\frac{1}{p_{k}}\left[1-e^{-d T_{s} p_{k}}\right] .
\end{aligned}
$$

The next step is to apply duHamel's formula over the interval $d T_{s} \leq t \leq T_{s}$ to derive an expression for $x_{k}(t)$ at the end of the switching period:

$$
\begin{aligned}
x_{k}\left(T_{s}\right) & =e^{-p_{k}(1-d) T_{s}} x_{k}\left(d T_{s}\right)+\int_{0}^{(1-d) T_{s}} e^{-\left((1-d) T_{s}-\xi\right) p_{k}} d \xi \\
& =e^{-p_{k} T_{s}} x_{k}(0)-\frac{1}{p_{k}}\left[2 e^{-p_{k}(1-d) T_{s}}-e^{-p_{k} T_{s}}-1\right] .
\end{aligned}
$$

By making use of the fact that $x_{k}(t)$ is periodic, i.e. $x_{k}\left(T_{s}\right)=x_{k}(0)$, it is now possible to calculate an expression for $x_{k}(0)$ :

$$
x_{k}(0)=\frac{2 e^{-p_{k}(1-d) T_{s}}-e^{-p_{k} T_{s}}-1}{p_{k}\left(e^{-p_{k} T_{s}}-1\right)} .
$$

Knowing the value of $x_{k}(0)$, it is possible to calculate the derivative of $x_{k}(t)$ just prior to $d T_{s}$ :

$$
\begin{aligned}
\lim _{\substack{t \rightarrow d T_{s} \\
t \leq d T_{s}}} \frac{d x_{k}(t)}{d t} & =-p_{k} e^{-p_{k} d T_{s}} x_{k}(0)-e^{-p_{k} d T_{s}} \\
& =2\left(\frac{e^{-p_{k} T_{s}}-e^{-p_{k} d T_{s}}}{1-e^{-p_{k} T_{s}}}\right) .
\end{aligned}
$$

Adding the contributions of all $K$ branches in the partial fraction expansion results in the following expression for the ripple gradient:

$$
\lim _{\substack{t \rightarrow d T_{s} \\ t \leq d T_{s}}}\left[\frac{d f(t)}{d t}\right]=2 \sum_{k=1}^{K} A_{k}\left(\frac{e^{-p_{k} T_{s}}-e^{-p_{k} d T_{s}}}{1-e^{-p_{k} T_{s}}}\right) .
$$




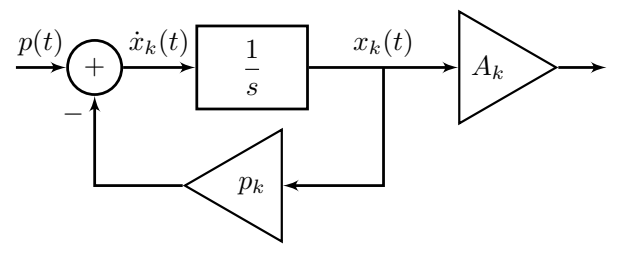

Figure 18: Block diagram representation of each of the terms of the partial fraction expansion of Eq. (5).

\section{REFERENCES}

[1] R. Andeen, "Analysis of pulse duration sampled-data systems with linear elements," IRE Transactions on Automatic Control, vol. 5, no. 4, pp. 306-313, Sep 1960.

[2] T. Kadota and H. Bourne, "Stability conditions of pulse-width-modulated systems through the second method of Lyapunov," IRE Transactions on Automatic Control, vol. 6, no. 3, pp. 266-276, Sep 1961.

[3] G. Murphy and S. Wu, "A stability criterion for pulse-width-modulated feedback control systems," IEEE Transactions on Automatic Control, vol. 9, no. 4, pp. 434-441, Oct 1964.

[4] R. Skoog and G. Blankenship, "Generalized pulse-modulated feedback systems: Norms, gains, Lipschitz constants, and stability," IEEE Transactions on Automatic Control, vol. 15, no. 3, pp. 300-315, Jun 1970.

[5] P. T. Krein and R. M. Bass, "Multiple limit cycle phenomena in switching power converters," in Applied Power Electronics Conference and Exposition, 1989. APEC' 89. Conference Proceedings 1989., Fourth Annual IEEE, Mar 1989, pp. 143-148.

[6] F. C. Lee and R. A. Carter, "Investigations of stability and dynamic performances of switching regulators employing current-injected control," in Power Electronics Specialists Conference, 1981 IEEE, June 1981, pp. 3-16.

[7] R. D. Middlebrook and S. Cuk, "A general unified approach to modelling switching-converter power stages," in Power Electronics Specialists Conference, 1976 IEEE, June 1976, pp. 18-34.

[8] Y. Yan, F. C. Lee, and P. Mattavelli, "Comparison of small signal characteristics in current mode control schemes for point-of-load buck converter applications," IEEE Transactions on Power Electronics, vol. 28, no. 7, pp. 3405-3414, July 2013.

[9] S. R. Sanders, J. M. Noworolski, X. Z. Liu, and G. C. Verghese, "Generalized averaging method for power conversion circuits," in Power Electronics Specialists Conference, 1990. PESC '90 Record., 21st Annual IEEE, 1990, pp. 333-340.

[10] B. Lehman and R. M. Bass, "Extensions of averaging theory for power electronic systems," in Power Electronics Specialists Conference, PESC '94 Record., 25th Annual IEEE, Jun 1994, pp. 1053-1057 vol.2.

[11] T. A. Sakharuk, B. Lehman, A. M. Stankovic, and G. Tadmor, "Effects of finite switching frequency and delay on pwm controlled systems," IEEE Transactions on Circuits and Systems I: Fundamental Theory and Applications, vol. 47, no. 4, pp. 555-567, Apr 2000.

[12] M. Cespedes and J. Sun, "Impedance modeling and analysis of grid-connected voltage-source converters," IEEE Transactions on Power Electronics, vol. 29, no. 3, pp. 1254-1261, March 2014.

[13] B. Wen, D. Boroyevich, P. Mattavelli, R. Burgos, and Z. Shen, "Impedance-based analysis of grid-synchronization stability for three-phase paralleled converters," in 2014 IEEE Applied Power Electronics Conference and Exposition - APEC 2014, March 2014, pp. $1233-1239$.

[14] S. Bacha, I. Munteanu, and A. Bratcu, Power Electronic Converters Modeling and Control: with Case Studies, ser. Advanced Textbooks in Control and Signal Processing. Springer London, 2013. [Online]. Available: https://books.google.co.za/books?id=ijW3BAAAQBAJ

[15] D. J. Packard, "Discrete modeling and analysis of switching regulators," Ph.D. dissertation, California Institute of Technology, 1976.

[16] Y. Yu, F. C. Y. Lee, and J. Kolecki, "Modeling and analysis of power processing systems," in Power Electronics Specialists Conference, 1979 IEEE, June 1979, pp. 11-24.

[17] A. R. Brown and R. Middelbrook, "Sampled-data modeling of switching regulators," in Power Electronics Specialists Conference, 1981 IEEE, June 1981, pp. 349-369.

[18] G. C. Verghese, M. E. Elbuluk, and J. G. Kassakian, "A general approach to sampled-data modeling for power electronic circuits," IEEE Transactions on Power Electronics, vol. PE-1, no. 2, pp. 76-89, April 1986. 
[19] C.-C. Fang and E. H. Abed, "Sampled-data modeling and analysis of closed-loop pwm dc-dc converters," in Circuits and Systems, 1999. ISCAS '99. Proceedings of the 1999 IEEE International Symposium on, vol. 5, 1999, pp. 110-115 vol.5.

[20] — - "Sampled-data modelling and analysis of the power stage of pwm dc-dc converters," International Journal of Electronics, vol. 88, no. 3, pp. 347-369, 2001. [Online]. Available: http://dx.doi.org/10.1080/00207210010004111

[21] M. M. Khan and W. Zhi-Ming, "A generalized framework for sampled-data model analysis of closed-loop pwm dc-dc converter system," in Industrial Electronics Society, 2001. IECON '01. The 27th Annual Conference of the IEEE, vol. 2, 2001, pp. 820-825 vol.2.

[22] D. Costinett, R. Zane, and D. Maksimovi, "Discrete-time small-signal modeling of a $1 \mathrm{MHz}$ efficiency-optimized dual active bridge converter with varying load," in Control and Modeling for Power Electronics (COMPEL), 2012 IEEE 13th Workshop on, June 2012, pp. $1-7$.

[23] L. Risbo, "Discrete-time modeling of continuous-time pulse width modulator loops," in Audio Engineering Society Conference: 27th International Conference: Efficient Audio Power Amplification, 9 2005. [Online]. Available: http://www.aes.org/elib/browse.cfm?elib=13263

[24] L. Risbo, M. C. W. Høyerby, and M. A. E. Andersen, "A versatile discrete-time approach for modeling switch-mode controllers," in 2008 IEEE Power Electronics Specialists Conference, June 2008, pp. 1008-1014.

[25] L. Risbo and M. C. W. Høyerby, "Suppression of continuous-time and discrete-time errors in switch-mode control loops," in Audio Engineering Society Conference: 37th International Conference: Class D Audio Amplification, Aug 2009. [Online]. Available: http://www.aes.org/e-lib/browse.cfm?elib=15221

[26] S. M. Cox and H. d. T. Mouton, "Ripple compensation for a class-d amplifier," SIAM Journal on Applied Mathematics, vol. 75, no. 4, pp. 1536-1552, 2015.

[27] B. Putzeys, "Simple, ultralow distortion digital pulse width modulator," in Audio Engineering Society Convention 120, 5 2006. [Online]. Available: http://www.aes.org/e-lib/browse.cfm?elib=13498

[28] T. Mouton, A. de Beer, B. Putzeys, and B. McGrath, "Modelling and design of single-edge oversampled pwm current regulators using z-domain methods," in ECCE Asia Downunder (ECCE Asia), 2013 IEEE, June 2013, pp. 31-37.

[29] D. Maksimovic, "Computer-aided small-signal analysis based on impulse response of dc/dc switching power converters," IEEE Transactions on Power Electronics, vol. 15, no. 6, pp. 1183-1191, Nov 2000.

[30] J. Nilsson and S. Riedel, Electric Circuits. Pearson/Prentice Hall, 2008.

[31] H. d. T. Mouton and B. Putzeys, "Understanding the PWM nonlinearity: Single-sided modulation," Power Electronics, IEEE Transactions on, vol. 27, no. 4, pp. 2116 - 2128, Apr. 2012.

[32] D. M. van de Sype, K. D. Gusseme, A. R. van den Bossche, and J. A. Melkebeek, "Small-signal z-domain analysis of digitally controlled converters," in Power Electronics Specialists Conference, 2004. PESC 04. 2004 IEEE 35th Annual, vol. 6, June 2004, pp. 4299-4305 Vol.6.

[33] D. Maksimovic and R. Zane, "Small-signal discrete-time modeling of digitally controlled dc-dc converters," in 2006 IEEE Workshops on Computers in Power Electronics, July 2006, pp. 231-235.

[34] L. Corradini, D. Maksimovic, P. Mattavelli, and R. Zane, Digital Control of High-Frequency Switched-Mode Power Converters. Wiley-IEEE Press, 2015. [Online]. Available: http://ieeexplore.ieee.org/xpl/articleDetails.jsp?arnumber=7199765

[35] G. Goodwin, S. Graebe, and M. Salgado, Control system design. Prentice Hall, 2001.

[36] D. Jordan and P. Smith, Nonlinear Ordinary Differential Equations: An Introduction for Scientists and Engineers, ser. Oxford applied and engineering mathematics. Oxford University Press, 2007.

[37] M. Abramowitz, Handbook of Mathematical Functions, With Formulas, Graphs, and Mathematical Tables,. Dover Publications, Incorporated, 1974.

[38] A. Oppenheim, W. Schafer, and J. Buck, Discrete-time signal processing. Prentice Hall, 1998.

[39] T. Mouton and B. Putzeys, "Digital control of a PWM switching amplifier with global feedback," in Audio Engineering Society Conference: 37th International Conference: Class D Audio Amplification, 8 2009. [Online]. Available: http://www.aes.org/e-lib/browse.cfm?elib=15218

[40] P. Kemp, "High-order analog control of a clocked class-d audio amplifier with global feedback using z-domain methods," Master's thesis, University of Stellenbosch, 2011. [Online]. Available: https://scholar.sun.ac.za/handle/10019.1/42918

[41] P. Kemp, T. Mouton, and B. Putzeys, "High-order analog control of a clocked class-d audio amplifier with global feedback using z-domain methods," in Audio Engineering Society Convention 131, Oct 2011. [Online]. Available: http://www.aes.org/e-lib/browse.cfm?elib=15994 
[42] C. Baesens, "Slow sweep through a period-doubling cascade: Delayed bifurcations and renormalisation," Physica D Nonlinear Phenomena, vol. 53, pp. 319-375, Nov. 1991.

[43] M. Taylor, Introduction to Differential Equations, ser. Pure and applied undergraduate texts. American Mathematical Society, 2011. 